\title{
Relação entre o estado nutricional materno e sobrepeso nas crianças brasileiras*
}

\section{Relationship between maternal nutritional status and overweight in Brazilian children}

\author{
Elyne M. Engstrom e Luiz A. Anjos \\ Secretaria Estadual de Saúde do Rio de Janeiro, PAISMCA. Rio de Janeiro, RJ - Brasil (E.M.E.), \\ Escola Nacional de Saúde Pública. Rio de Janeiro, RJ - Brasil (L.A.A.)
}

\begin{abstract}
Resumo
Investigou-se a relação entre o estado nutricional de mães $(n=6.289)$ e sobrepeso nas crianças brasileiras menores de 10 anos $(n=14.914)$, considerando-se a influência de condições sociais num inquérito nutricional da população brasileira realizado em 1989, a Pesquisa Nacional sobre Saúde e Nutrição. $\mathrm{O}$ estado nutricional materno, segundo o índice de massa corporal (IMC, $\mathrm{kg}$ / $\mathrm{m}^{2}$ ), foi classificado em: baixo peso (BP; IMC $\left.<20\right)$; peso adequado ( $20 \leq$ IMC $<25$ ) e sobrepeso (SP; IMC $\geq 25$ ). Para sobrepeso nas crianças, utilizou-se o indicador peso para estatura $(\mathrm{P} / \mathrm{E}) \geq+2$ desvios-padrão da curva de crescimento da população americana. A prevalência de SP nas crianças foi de $4,8 \%$ e nas mães houve $15,3 \%$ de BP e 35,9\% de SP. Tomando-se as mães com baixo peso como referência, as crianças com sobrepeso tiveram maior chance de ter mães também com sobrepeso (odds ratio; $\mathrm{OR}=3,19 ; 95 \%$ intervalo de confiança (IC $=2,24-4,53$ ), sendo o $\mathrm{OR}=2,46$ (IC $=1,73-3,50)$ para as mães com estado nutricional adequado. Sobrepeso nas crianças foi influenciado diretamente pela escolaridade materna $(\mathrm{OR}=2,89$; $\mathrm{IC}=1,74-4,80$ para mães com $\geq 12$ séries cursadas em referência às mães analfabetas); pela renda domiciliar per capita $(\mathrm{OR}=3,82$; IC $=2,79-5,22$ nas mães no quarto quartil em comparação ao primeiro quartil) e pelas condições de moradia $(\mathrm{OR}=2,69$; $\mathrm{IC}=2,05-3,54$ nas mães de domicílios de boas condições em relação àquelas de piores condições de moradia). Os resultados sugerem haver relação direta entre o estado nutricional materno e sobrepeso nas crianças brasileiras.
\end{abstract}

Estado nutricional. Obesidade, etiologia.

\begin{abstract}
The relationship between maternal (age between 20 and 50 years) nutritional status (NS) and children's (age < 10 years) obesity level was studied in a probabilistic sample of the Brazilian population of all ages $(n=63,213)$ conducted in 1989. Maternal NS was classified as underweight (UW - Body mass index $\left.(B M I)<20 \mathrm{~kg} \cdot \mathrm{m}^{-2}\right)$, normal $(20 \leq B M I<25)$ and overweight $(O W$, $B M I \leq 25)$. Overweight was identified in children with body mass of stature
\end{abstract}

\footnotetext{
* Financiado parcialmente pelo Conselho Nacional de Desenvolvimento Científico e Tecnológico/CNPq (Processo nº 301076/89-8 e 132534/92-4). Baseado na Dissertação de Mestrado intitulada Inter-relação do estado nutricional e condições socioambientais do binômio mãe-filho, Brasil, 1989, apresentada ao Instituto Fernandes Figueiras da Fundação Oswaldo Cruz, em 1994. Apresentado no $7^{\circ}$ Congresso Internacional de Obesidade, Toronto, Canadá, 1994.

Correspondência para / Correspondence to: Luiz Antonio dos Anjos - Escola Nacional de Saúde Pública. Fundação Oswaldo Cruz. R. Leopoldo Bulhões, 1.480 - Manguinhos. 21041-210 Rio de Janeiro, RJ - Brasil. Fax: (021) 270.3219 E-mail: ANJOS@ DCCØØ1.CICT.FIOCRUZ.BR Recebido em 10.10.1994. Representado em 28.11.1995. Aprovado em 15.2.1996.
} 
$\geq+2$ standard deviations of the American growth reference curve (NCHS). There were a total of 12,414 mother-child pairs. OW was observed in $4.8 \%$ of the children. $U W$ and $O W$ were seen in 15.3 and $35.9 \%$ of the mothers, respectively. With the UW mothers as reference, the odds ratio (OR) of a child being obese and his/her mother's NS being normal or OW was 2.5 (95\% confidence interval (CI), 1.7-3.5) and 3.2 (2.2-4.5), respectively. This relationship was influenced by maternal education, $O R=2.9(1.7-4.8)$ for mothers with 12 years of education in relation of the ones without any formal education and family percapita income, $O R=3.8(2.8-5.2)$ for mothers in the $4^{\text {th }}$ in relation to those in the $1^{\text {st }}$ quartile. These data suggest a positive relationship between maternal and child obesity in the Brazilian population.

Nutritional status. Obesity, etiology.

\section{INTRODUÇÃO}

O estado nutricional materno durante a gestação é de importância fundamental no peso ao nascer da criança $^{8,11}$, já que boas condições do ambiente uterino favorecerão o desenvolvimento fetal adequado. Todavia, saindo do período neonatal e considerando-se a relação do estado nutricional das mães e seus filhos durante a infância, observa-se carência de estudos, especialmente em países em desenvolvimento.

Particularmente no que se refere à obesidade, os estudos em países desenvolvidos têm apontado para uma associação familiar, ou seja, as crianças cujos pais apresentam sobrepeso ou obesidade têm maior chance de também o terem ${ }^{9,15,17}$. Nesses países, a obesidade é considerada importante problema de saúde pública, associando-se, na população adulta, a importante morbi-mortalidade ${ }^{5}$. A abordagem preventiva da obesidade dirige-se, atualmente, ao grupo etário infantil, visto que o processo patológico com início na infância, pode se perpetuar na vida adulta, dificultando seu controle ${ }^{10,19}$.

$\mathrm{Na}$ relação familiar para a obesidade, discute-se a importância de condições genéticas e ambientais como principais determinantes dessa alteração nutricional $^{3}$. A expressão fenotípica de alguns gens está intimamente relacionada a fatores ambientais, em uma constante interação genética-ambiente ${ }^{31}$. Pode-se esperar que as crianças e suas mães, ao compartilharem condições socioambientais-culturais semelhantes, estejam associadas diretamente em seu estado nutricional.

Na população adulta brasileira, os dados de um inquérito nacional conduzido em 1989, a Pesquisa Nacional sobre Saúde e Nutrição (PNSN), evidenciaram prevalência de $39,4 \%$ de sobrepeso e obesidade em mulheres ${ }^{2}$, mostrando ser um problema nutricional de grandes proporções em nosso meio. Com base nos dados do citado inquérito, o presente trabalho analisou a associação entre o sobrepeso em crianças brasileiras menores de 10 anos com o estado nutricional materno, percebendo a relativa influência de determinadas variáveis socioambientais no estado nutricional do binômio mãe-filho.

\section{MATERIAL E MÉTODO}

Para elaboração do presente estudo, foram utilizadas informações coletadas pela $\mathrm{PNSN}^{*}$, um inquérito nutricional brasileiro, realizado em 1989, cujo objetivo central foi a aferição do estado de saúde e nutrição da população. A pesquisa utilizou uma amostragem complexa, de base domiciliar, tendo como âmbitos de análise as cinco grandes regiões geográficas brasileiras (Norte, Nordeste, Sudeste, Sul e Centro-Oeste) e as situações urbana e rural de cada região, exetuando-se o Norte rural, por problemas operacionais, o que totalizou 9 âmbitos de análise ${ }^{13}$.

Na primeira etapa da amostragem da PNSN, os 9 âmbitos da pesquisa foram estratificados segunda a renda domiciliar média do setor demográfico e a taxa de alfabetização da população de 5 a 15 anos, com base em estimativas feitas a partir do censo nacional de 1980. Para cada estrato foram sorteados setores censitários, tendo o domicílio como unidade amostral primária, com cada setor constituindo-se em um aglomerado. Em uma segunda fase, a média de 36 domicílios foi sorteada para cada setor, totalizando 14.455 domicílios investigados, com dados de 63.213 brasileiros de todas as idades ${ }^{14}$.

Além das medidas antropométricas de massa corporal e estatura, núcleo central da PNSN, foram coletadas, por meio de questionários padronizados, informações sobre características demográficas básicas como renda, condições de habitação, instrução, ocupação, dentre outras.

* O levantamento dos dados da Pesquisa Nacional sobre Saúde e Nutrição (PNSN-1989) foi patrocinado pelo Instituto Nacional de Alimentação e Nutrição (INAN) e executado com colaboração técnica da Fundação Instituto Brasileiro de Geografia e Estatística (IBGE) e do Instituto de Planejamento Econômico e Social (IPEA). As análises e interpretações aqui apresentadas são de inteira responsabilidade dos autores e não indicam aprovação por parte das instituições acima referidas. 
Todas as informações foram coletadas nos domicílios dos sujeitos, por equipe treinada.

A casuística do presente trabalho compreendeu todas as mães com idade entre 20 e 50 anos, que tinham ao menos um filho menor de 10 anos, no total de 6.289 mães. Utilizou-se para classificação do estado nutricional materno o índice de massa corporal (IMC), que parece ser um bom indicador nutricional em estudos epidemiológicos em adultos ${ }^{1}$. O IMC é calculado pela razão entre massa corporal e estatura ao quadrado, com os seguintes pontos de corte $^{37}$ : Baixo Peso (IMC < 20); Adequado ( $20 \leq$ IMC $<25$ ) e Sobrepeso (IMC $\geq 25$ ).

Para as crianças, a casuística compreendeu 14.914 menores de 10 anos, de ambos os sexos. O estado nutricional das crianças foi classificado pelo Índice peso/ estatura (P/E), expresso em valor $\mathrm{Z}$ da mediana da população de referência americana ${ }^{23}$, segundo recomendação da Organização Mundial da Saúde ${ }^{34,36}$. Utilizou-se como ponto de corte para sobrepeso ${ }^{35}$, o valor de $\mathrm{ZP} / \mathrm{E} \geq+2$.

As variáveis sociais estudadas compreenderam: 1) renda domiciliar per capita, expressa em quartis de distribuição nacional; 2) escolaridade materna, avaliada segundo a capacidade de ler e escrever, o grau e a última série escolar cursada pela mãe, resultando em 6 faixas: escolaridade zero/analfabeta, escolaridade zero mas sabe ler e escrever, 1-4 séries, 5 -8, 9-11 e $\geq 12$ séries cursadas; 3) condições de moradia, avaliando o ambiente físico domiciliar, sendo reunidas em um indicador proposto por Bronfman e col. ${ }^{4}$ (1988) e adaptado para a PNSN por Ribeiro $^{26}(1994)$. Este indicador considerou o piso do domicílio, abastecimento de água, saneamento e número de moradores por cômodo, com 3 categorias finais: boa, regular ou má moradia. Quando cruzaram-se os dados de estado nutricional de mães e filhos com as variáveis sociais houve redução de amostra por ausência das informações maternas e sociais. Portanto, o número total de casos nas Tabelas varia segundo o cruzamento em questão.

$\mathrm{Na}$ análise da relação do estado nutricional entre mães e filhos, a medida de associação utilizada foi expressa em odds ratio (OR), segundo regressão logística ${ }^{18}$, sendo a variável dependente o estado nutricional infantil e as demais variáveis como independentes. O OR é uma medida de associação diretamente estimada através de um modelo de regressão logística. Ao comparar as frequiências de cada variável com uma referência previamente determinada possibilita identificar os grupos de maior risco de apresentar a "doença" (no caso do presente estudo sobrepeso). Em um estudo transversal como a PNSN, o risco aqui referido não implica em causalidade, ou seja, não associa causa/efeito entre as variáveis estudadas e sim uma associação entre as variáveis.

O OR foi calculado pelo exponencial do coeficiente beta A variável que apresentava a menor freqüiência foi considerada como a referência $(O R=1,0)$ na regressão logística. Utilizou-se para análise dos dados, o software SUDDAN versão 6.34 (Survey Data Analysis for Multistage Samples Design), que incorpora em cada uma das análises, o erro amostral no efeito de desenho (“deff”), ajustando os cálculos das freqüências ${ }^{29}$.

\section{RESULTADOS}

Observou-se nas 14.914 crianças estudadas, a prevalência de $4,8 \%$ de sobrepeso, correspondendo a um total de 618 crianças menores de 10 anos. Quanto ao estado nutricional materno, evidenciou-se prevalência de $15,3 \%$ de baixo peso, $35,9 \%$ de sobrepeso, sendo que $48,8 \%$ das mães tinham o IMC adequado. A prevalência de sobrepeso foi semelhante entre sexo (4,5 e 4,9\% para meninos e meninas, respectivamente).

A prevalência de sobrepeso nas crianças, segundo a escolaridade materna, foi menor nas mães com nenhuma ou pouca escolaridade (3,3\% até 4 séries cursadas), aumentando conforme o aumento da escolaridade, com maior prevalência na faixa de maior escolaridade, ou seja, nas mães com 12 ou mais séries cursadas $(9,0 \%$, Tabela 1$)$. O risco da criança ter sobrepeso foi 2,89 vezes maior se a mãe tinha mais escolaridade, em relação a mãe analfabeta ou com 1-4 séries, tidas como referência.

Quanto à renda domiciliar per capita, as crianças do quartil de renda mais alto ( $4^{\circ}$ quartil) tiveram maior prevalência de sobrepeso $(10,4 \%)$. A OR foi de 3,82 no $4^{\circ}$ quartil, em relação ao $1^{\circ}$ quartil, sendo que $2^{\circ} \mathrm{e}$ $3^{\circ}$ tiveram prevalência de sobrepeso e OR bastante semelhantes. Para as condições de moradia, observouse aumento de sobrepeso, conforme a melhoria dessas condições, com $2,7 \%$ nas crianças dos domicílios em más condições (a referência) e 6,9\% nas de boas condições de moradia ( $\mathrm{OR}=2,69$, Tabela 1$)$.

Já entre as mães, a relação de sobrepeso e variáveis sociais mostrou, quanto à escolaridade materna, maior prevalência de sobrepeso naquelas que sabiam ler e escrever, mas não tinham escolaridade formal, e de 1 -4 séries cursadas $(38,4$ e $38,8 \%$, respectivamente), havendo menor prevalência nas mulheres de maior escolaridade $(29,8 \%$, Tabela 2$)$. Quanto à prevalência de sobrepeso por renda, observou-se relação direta até o $3^{\circ}$ quartil, decaindo no grupo mais privilegiado, sendo $28,2 \%$ no $1^{\circ}$ quartil e $36,6 \%$ no $4^{\circ}$ quartil de renda domiciliar per capita. Para as condições de moradia, a freqüência foi maior nos domicílios de condições regular ou boa $(41,8 \mathrm{e}$ $40,7 \%$, respectivamente), sendo de $26,5 \%$ nos domicílios de piores condições.

A relação entre estado nutricional materno e sobrepeso nas crianças foi analisada para um total de 12.414 crianças menores de 10 anos. Observou-se que das 3.914 crianças que tinham mães com sobrepeso, 244 crianças $(6,2 \%)$ tinham sobrepeso (Tabela 3). A freqüência diminui para $2,0 \%$ se a mãe tinha baixo peso, ou seja, do total de 2.135 crianças cujas mães 
Tabela 1 - Prevalência de sobrepeso (Z-P/E $\leq+2)$ em crianças brasileiras por escolaridade materna, quartil de renda domiciliar per capita e condições de moradia.*

\begin{tabular}{|c|c|c|c|c|c|}
\hline & \multirow{2}{*}{$\begin{array}{c}\text { Total } \\
\mathrm{N}\end{array}$} & \multicolumn{2}{|c|}{$\begin{array}{c}\text { Crianças com } \\
\text { sobrepeso }\end{array}$} & \multicolumn{2}{|c|}{ Odds ratio (IC) } \\
\hline & & $\mathrm{N}$ & $\%$ & & \\
\hline \multicolumn{6}{|c|}{ Escolaridade materna (séries) } \\
\hline 0 - Analfabeta & 2.365 & 62 & 3,3 & 1,00 & - \\
\hline 0 - Lê e escreve & 238 & 6 & 1,6 & 0,47 & $(0,17-1,30)$ \\
\hline $1-4$ & 5.315 & 163 & 3,3 & 1,00 & - \\
\hline $5-8$ & 2.403 & 134 & 6,3 & 1,95 & $(1,35-2,83)$ \\
\hline $9-11$ & 1.420 & 109 & 7,6 & 2,39 & $(1,52-3,74)$ \\
\hline$\geq 12$ & 590 & 50 & 9,0 & 2,89 & $(1,73-4,80)$ \\
\hline Total & 12.331 & 524 & 4,8 & & \\
\hline \multicolumn{6}{|c|}{ Renda domiciliar per capita } \\
\hline Primeiro quartil & 4.378 & 110 & 2,4 & 1,00 & - \\
\hline Segundo quartil & 3.475 & 126 & 4,5 & 1,52 & $(1,05-2,20)$ \\
\hline Terceiro quartil & 2.180 & 97 & 4,3 & 1,48 & $(0,99-2,19)$ \\
\hline Quarto quartil & 1.883 & 186 & 10,4 & 3,82 & $(2,79-5,22)$ \\
\hline Total & 11.916 & 519 & 4,9 & & \\
\hline \multicolumn{6}{|c|}{ Condições de moradia } \\
\hline Má & 6.658 & 176 & 2,7 & 1,00 & - \\
\hline Regular & 2.969 & 110 & 3,4 & 1,26 & $(0,82-1,94)$ \\
\hline Boa & 3.798 & 256 & 6,9 & 2,69 & $(2,05-3,54)$ \\
\hline Total & 13.425 & 542 & 4,5 & & \\
\hline
\end{tabular}

* Dados da Pesquisa Nacional sobre Saúde e Nutrição (PNSN), 1989.

IC - Intervalo de confiança

Tabela 2 - Prevalência de baixo peso e sobrepeso em mães brasileiras em função da escolaridade, quartil de renda domiciliar per capita e condições de moradia.*

\begin{tabular}{|c|c|c|c|c|c|}
\hline & \multirow{2}{*}{$\begin{array}{c}\text { Total } \\
\mathrm{N}\end{array}$} & \multicolumn{2}{|c|}{ Baixo peso $(\mathrm{IMC}<20)$} & \multicolumn{2}{|c|}{ Sobrepeso $(\mathrm{IMC} \geq 25)$} \\
\hline & & $\mathrm{N}$ & $\%$ & $\mathrm{~N}$ & $\%$ \\
\hline \multicolumn{6}{|c|}{ Escolaridade materna (séries) } \\
\hline 0 - Analfabeta & 995 & 203 & 18,7 & 303 & 31,7 \\
\hline 0 - Lê e escreve & 106 & 19 & 19,5 & 33 & 38,4 \\
\hline $1-4$ & 2.562 & 414 & 15,3 & 913 & 38,8 \\
\hline $5-8$ & 1.335 & 194 & 12,2 & 472 & 37,0 \\
\hline $9-11$ & 879 & 135 & 16,1 & 292 & 34,2 \\
\hline$\geq 12$ & 370 & 48 & 15,5 & 105 & 29,8 \\
\hline Total & 6.247 & 1.013 & 15,3 & 2.118 & 36,1 \\
\hline \multicolumn{6}{|c|}{ Renda domiciliar per capita } \\
\hline Primeiro quartil & 1.729 & 380 & 22,2 & 502 & 28,2 \\
\hline Segundo quartil & 1.771 & 292 & 14,6 & 603 & 37,5 \\
\hline Terceiro quartil & 1.375 & 161 & 12,4 & 524 & 41,9 \\
\hline Quarto quartil & 1.225 & 145 & 11,8 & 450 & 36,6 \\
\hline Total & 6.100 & 978 & 15,3 & 2.079 & 36,1 \\
\hline \multicolumn{6}{|c|}{ Condições de moradia } \\
\hline Má & 2.224 & 485 & 22,5 & 614 & 26,5 \\
\hline Regular & 1.415 & 194 & 11,3 & 549 & 41,8 \\
\hline Boa & 2.041 & 223 & 11,7 & 804 & 40,7 \\
\hline Total & 5.680 & 902 & 14,8 & 1.967 & 36,8 \\
\hline
\end{tabular}

* Dados da Pesquisa Nacional sobre Saúde e Nutrição (PNSN), 1989.

IMC - Índice de massa corporal. 
Tabela 3 - Prevalência de sobrepeso nas crianças, segundo peso para estatura (Z-P/E $\geq+2)$ em relação ao estado nutricional materno.*

\begin{tabular}{|c|c|c|c|c|c|c|}
\hline \multirow[t]{2}{*}{ Estado nutricional materno } & \multicolumn{2}{|c|}{$\begin{array}{c}\text { Crianças com } \\
\text { sobrepeso }(\geq \mathrm{P} / \mathrm{E} \geq+2)\end{array}$} & \multicolumn{2}{|c|}{$\begin{array}{c}\text { Crianças sem } \\
\text { sobrepeso }(\geq \mathrm{P} / \mathrm{E}<+2)\end{array}$} & \multicolumn{2}{|c|}{ Total } \\
\hline & $\mathrm{N}$ & $\%$ & $\mathrm{~N}$ & $\%$ & $\mathrm{~N}$ & $\%$ \\
\hline Baixo peso & 42 & 2,0 & 2.093 & 98,0 & 2.135 & 100,0 \\
\hline Adequado & 242 & 4,8 & 6.123 & 95,2 & 6.365 & 100,0 \\
\hline Sobrepeso & 244 & 6,2 & 3.670 & 93,8 & 3.914 & 100,0 \\
\hline Total & 528 & 4,8 & 11.886 & 95,2 & 12.414 & 100,0 \\
\hline
\end{tabular}

Baixo peso $=\mathrm{IMC}<20$

Adequado $=(20 \leq \mathrm{IMC}<25)$

Sobrepeso $=I M C \geq 25$

* Dados da Pesquisa Nacional sobre Saúde e Nutrição (PNSN)

tinham baixo peso, apenas 42 tinham sobrepeso, encontrando-se, ainda, $4,8 \%$ de crianças com sobrepeso nas mães de estado nutricional adequado.

A associação do estado nutricional de mães e filhos demonstrou que o OR para a relação criança/mãe, com sobrepeso, foi de 3,19, tomando-se como referência as crianças com sobrepeso/mães com baixo peso. O OR foi de 2,46 para as crianças com sobrepeso/ mães com estado nutricional adequado (Tabela 4).

Tabela 4 - Odds ratio (OR) e intervalo de confiança de $95 \%$ (IC) da relação entre estado nutricional materno e o sobrepeso infantil.*

\begin{tabular}{lrc}
\hline Estado nutricional materno & OR & IC 95\% \\
\hline Baixo peso & 1,0 & - \\
Adequado & 2,46 & $(1,73-3,50)$ \\
Sobrepeso & 3,19 & $(2,24-4,53)$ \\
\hline
\end{tabular}

* Dados da Pesquisa Nacional sobre Saúde e Nutrição (PNSN)

IC - Intervalo de confiança

\section{DISCUSSÃO}

O presente estudo evidenciou que mães e filhos brasileiros têm um relação direta em seu estado nutricional, no que se refere ao sobrepeso. $\mathrm{O}$ risco de uma criança ter sobrepeso foi 3,19 vezes maior se a mãe também apresentava sobrepeso, em relação à mãe com baixo peso. Essa associação enfatiza a necessidade da abordagem familiar para o sobrepeso e a obesidade, detectando precocemente crianças com tendência à obesidade ${ }^{10,25}$.

O conhecimento no campo da epidemiologia, apontando para aqueles grupos com maior chance de desenvolver tal alteração nutricional, pode trazer importantes contribuições na abordagem clínica da obesidade. Sabe-se da dificuldade de tratar os adultos obesos, que têm associação com patologias de importante morbi-mortalidade no mundo moderno, cabendo agir preventivamente em relação ao grupo etário infantil. Obesidade na infância pode ser considerada preditiva de obesidade na vida adulta ${ }^{6,7}$. Serdula e col..$^{28}$ (1993) concluíram que as crianças americanas consi- deradas obesas tiveram 2 a 6,5 vezes mais chance de se tornarem adultos obesos, principalmente aquelas situadas nos níveis mais extremos de obesidade e em idades mais avançadas, como nos escolares.

Todavia, o sobrepeso e a obesidade não são alterações nutricionais restritas aos países desenvolvidos, onde se descrevem prevalências entre 5 e $25 \%$ de sobrepeso em crianças e adolescentes ${ }^{9}$. Em publicação preliminar anterior sobre PNSN, Taddei ${ }^{32}$ (1993) descreveu prevalência de 7\% de obesidade em meninos e $9 \%$ em meninas menores de 10 anos, utilizando o indicador massa corporal para estatura maior que $120 \%$ da mediana da população de referência americana $\left(\mathrm{NCHS}^{23}, 1977\right)$ encontrando maior percentual de crianças obesas em família de maior renda. O Brasil, apesar de possuir grandes contingentes de crianças com desnutrição crônica ${ }^{21}$, apresenta uma polarização em seu quadro nutricional, com prevalência elevada de sobrepeso na população adulta, como visto nesse estudo $(35,9 \%)$, podendose considerar problema de grandes proporções na população adulta brasileira. As crianças, analisadas em seu conjunto, demonstram prevalência de 4,8\% de sobrepeso, ainda bastante inferior ao grande problema nutricional do País, a desnutrição energéticoprotéica. No entanto, há que se detectar os grupos que apresentam, de modo particular, maior tendência ao sobrepeso. A inserção social das crianças e suas mães e a qualidade do ambiente vivenciado são importantes condições que determinam o estado de saúde e nutrição, devendo ser considerado ao se discutir sobrepeso e obesidade.

Com esse intuito, a criança não pode ser vista como uma unidade isolada, seu estado nutricional solitariamente avaliado, sem perceber o indivíduo inserido em seu contexto familiar, interagindo com seu meio ambiente. A figura materna surge como importante elo de ligação criança-ambiente, estabelecendo-se uma íntima relação mãe-filho desde a gestação, evoluindo durante a infância, rumo a independência biológica e social da criança. No âmbito familiar, mãe e filhos 
compartilham condições socioambientais semelhantes, com hábitos alimentares associados também aos aspectos culturais de cada grupo social, favorecendo uma relação direta em seu estado nutricional.

As variáveis sociais consideradas no presente estudo tiveram uma relação direta com o sobrepeso das crianças, embora com distribuição mais complexas quando analisado o estado nutricional materno. Isso pode ser entendido ao se observar que a prevalência de sobrepeso nas crianças menores de 10 anos aumentou segundo seu quartil de renda (Tabela 1). Do mesmo modo, a educação materna e as condições de moradia tiveram associação direta com sobrepeso nas crianças (e distribuição complexa quanto ao estado nutricional materno).

Estudos em países desenvolvidos demonstram que o nível socioeconômico está inversamente associado à obesidade nas mulheres adultas ${ }^{12,30} \mathrm{e}$ diretamente associado à obesidade nas crianças ${ }^{15,16}$. De modo contrário, Rolland-Cachera e Billesle ${ }^{27}$ (1986) evidenciaram maior prevalência de obesidade nas crianças francesas das classes trabalhadoras. Por sua vez, Sobal e Stunkard ${ }^{30}$ (1985), em revisão da literatura, encontraram maior prevalência de obesidade nas crianças de melhor nível socioeconômico sendo, nesse estrato, menor a prevalência de obesidade nas mulheres dos países desenvolvidos; no entanto, para aqueles em desenvolvimento, houve uma relação direta entre nível socioeconômico-obesidade nas mulheres. No caso brasileiro, nas mães estudadas há relação direta até o $3^{\circ}$ quartil de renda, porém com queda da prevalência de sobrepeso no estrato mais privilegiado. Num estudo longitudinal de todas as crianças nascidas em Pelotas (Brasil) durante um ano, Victora e col. ${ }^{33}$ (1988) referem que as crianças de nível socioeconômico mais alto tiveram maior prevalência de sobrepeso, em relação às crianças de nível mais baixo.

O acesso à educação, formal ou informal, contribui na melhoria do estado de saúde e nutrição dos indivíduos, articulando-se com outros fatores do meio

\section{REFERÊNCIAS BIBLIOGRÁFICAS}

1. ANJOS, L. A. Índice de massa corporal (massa corporal.estatura-2) como indicador do estado nutricional de adultos: uma revisão da literatura. Rev. Saúde Pública, 26: 431-6, 1992.

2. ANJOS, L. A. Valores antropométricos da população adulta brasileira: resultados da pesquisa nacional sobre saúde e nutrição. Rio de Janeiro, 1993. [Tese para obtenção do título de Professor Titular-Universidade Federal Fluminense]. social, permitindo a melhoria das condições de vida, de trabalho, de renda e, conseqüentemente, do ambiente físico do domicílio e acesso à alimentação. $\mathrm{O}$ nível de educação mostrou forte correlação negativa para a obesidade (mais importante até do que a renda) no estudo de Flegal e col. ${ }^{12}$ (1988), ao analisar os inquéritos nutricionais americanos de 1960 e 1980. No caso das mães brasileiras, a relação parece bem complexa. Há um aumento inicial com redução posterior, sendo que a prevalência de sobrepeso das mães analfabetas é semelhante a das mães com instrução de 12 anos ou mais. Entretanto, a relação sobrepeso em crianças e escolaridade materna é positiva.

A presença de saneamento básico, o tipo de moradia, o número de pessoas que habitam por cômodo da casa refletem a inserção social das famílias e relacionam-se a déficits nutricionais em estudos em países não desenvolvidos ${ }^{20,22,24}$. Seguindo a mesma lógica, pode-se esperar que haja maior prevalência de sobrepeso nos domicílios em boas condições de moradia, do que naqueles em más condições físicas. No presente estudo observou-se maior prevalência de sobrepeso tanto em crianças quanto em suas mães, nos domicílios de melhores condições.

Em suma, os dados da PNSN evidenciam uma relação direta de sobrepeso no binômio mãe-filho. Há que se valorizar, portanto, a monitorização contínua do estado nutricional das crianças, das gestantes, da população em todas as idades, não somente sob o aspecto da desnutrição energético-protéica assim como o sobrepeso. Esta vigilância nutricional permitirá oferecer subsídios para tomada de decisões nas políticas públicas, assim como avaliar a eficácia das ações empreendidas, visando a melhoria no estado de saúde e nutrição da população brasileira.

\section{AGRADECIMENTOS}

À Inês Rugani Ribeiro de Castro e, principalmente, aos revisores da Revista de Saúde Pública pelas valiosas sugestões.
3. BOUCHARD, C. Current understanding of the etiology of obesity: genetic and nongenetic factors. Am. J. Clin. Nutr., 53: $1561 \mathrm{~s}-5 \mathrm{~s}, 1991$.

4. BRONFMAN, M.; GUISCAFRE, H. P.; CASTRO, V.; CASTRO, R.; GUITIERREZ, G. II. La medición de la desiguald; una estrategia metodológica, analisis de las características socioeconómicas de la muestra. Arq. Invest. Méd., 19:351-60, 1988. 
5. BRAY, G. A. Pathophysiology of obesity. Am. J. Clin. Nutr., 55:488s-94s, 1992.

6. CASEY, V. A.; DWYER, J. T.; COLEMAN, K.A.; VALADIAN, I. Body mass index from childhood to middle age: a 50-y follow-up. Am. J. Clin. Nutr; 56:14-8, 1992.

7. CHARNEY, E.; GOODMAN, H. C.; MCBRIDGE, M.; LYON, B.; PRATT, R. Childhood antecedents of adult obesity. Do chubby infants become obese adults? N. Engl. J. Med., 295:6-9, 1976.

8. CUSMINSKY, M. \& OJEDA, E. N. S. Crecimiento y desarrollo: salud del niño y calidad de vida del adulto. In: Crecimiento y desarrollo: hechos y tendencias. Washington, D. C., Organización Panamericana de la Salud, 1988. (OPAS - Public. Cient., 519) p. 3-19.

9. DIETZ, W. H. Childhood obesity: susceptibility, cause and mangement. J. Pediatr., 103:676-86, 1983.

10. DIETZ, W. H. Critical periods in childhood for the development of obesity. Am. J. Clin. Nutr., 59:955-9, 1994.

11. EVELETH, P. G. Population differences in growth: environmental and genetic factors. In: Falkner, F. \& Tanner, M., org. Human growth. New York, Plenum Press, 1986. p. 221-39.

12. FLEGAL, K. M.; HARLAM, W. H.; LANDIS, J. R. Secular trends in body mass index and skinfold thickness with socioeconomic factors in young adult women. Am. J. Clin. Nutr., 48:535-43, 1988.

13. FLETCHER, P.R. Pesquisa nacional sobre saúde e nutrição: plano de amostragem. Brasília, OIT/PNUD/IPLAN/IPEA, 1987.

14. FLETCHER, P.R. Pesquisa nacional sobre saúde e nutrição: a seleção dos elementos da amostra na segunda etapa de amostragem. Brasília, OIT/PNUD/IPLAN/IPEA, 1988.

15. GARN, S. M. \& CLARK, D. C. Trends in fatness and the origins of obesity. Pediatrics, 57:443-56, 1976.

16. GARN, S. M.; HOPKINS, P. J.; RYAN, A. S. Differential fatness gain of low income boys and girls. Am. J. Clin. Nutr., 34:1465-8, 1981.

17. GARN, S. M.; SULLIVAN, T. V.; HAWTHORNE, V. M. Educational level, fatness and fatness differences between husbands and wives. Am. J. Clin. Nutr., 50:740-5, 1989.

18. KLEINBAUN, D. G.; KUPPER, L. L.; MORGENSTERN, H. Epidemiologic research. Belmont. Lifetime Learning Publications, 1982.

19. KWITTEROVICH, P. O. Diagnosis and management of familial dyslipoproteinemia in children and adolescents. Pediatr. Clin. N. Am., 37:1489-592, 1990.

20. MARTORELL, R.; MENDOZA, F.; CASTILLO, R. Poverty and stature in children. In: Waterlow, J. C., ed. Linear growth retardation in less developed countries. New York, Raven Press, 1988. (Nestlé Nutrtion Workshop Series, 14) p. 57-73.

21. MONTEIRO, C. A.; BENÍCIO, M. H. D.; GOUVEIA, N. C. Saúde e nutrição das crianças brasileiras no final da década de 80. In: Fundação IBGE. Perfil estatístico de crianças e mães no Brasil: aspectos de saúde e nutrição de crianças no Brasil, 1989. Rio de Janeiro, IBGE/UNICEF/IANAN, 1992. p. 19-42.
22. NABARRO, D.; HORWARD, P.; CASSELS, C.; PANT, M.; WIJGA, A.; PADFIELDN. The importance of infections and environmental factors as possible determinants of growth retardation in children. In: Waterlow, J. C.; ed. Linear growth retardation in less developed countries. New York, Raven Press, 1988. (Nestlé Nutrition Workshop Series, 14) p. 165-83.

23. NCHS. Growth curves for children birth-18 years. Vital Health Stat. Ser. 11 (165), 1977.

24. PRICE, R. A.; STUNKARD, A. J.; NESS, R.; WADDEN, T.; HESHKA, S.; KANDERS, B.; CORMILLOT, A. Childhood onset $($ age $<10)$ obesity has high familial risk. Int. J. Obes., 14: 185-95, 1990.

25. OLINTO, M. T.; VICTORA, C. G.; BARROS, F. C.; TOMASI, E. Determinantes da desnutrição infantil em uma população de baixa renda: um modelo de análise hierarquizado. Cad. Saúde Pública, 9(Supl. 1):14-27, 1993.

26. RIBEIRO, F. S. N. Da identidade do pesquisado à identidade da pesquisa: os trabalhadores brasileiros na pesquisa nacional sobre saúde e nutrição, 1994. [Dissertação de Mestrado - Escola Nacional de Saúde Pública].

27. ROLLAND-CACHERA, M. F. \& BELLISLE, F. No correlation between adiposity and food intake: why are working class children fatter? Am. J. Clin. Nutr., 44:779-87, 1986.

28. SERDULA, M. K.; IVERY, D.; COATES, R. J.; FREEDMAN, D. S.; WILLIAMSON, D. F.; BYERS, T. Do obese children become obese adults? A review of the literature. Prev. Med., 22: 167-77, 1993.

29. SHAH, B. V.; BRANWEL, B. G.; HUNT, P. N.; LAVANGE, L. M. SUDDAN user's manual: professional software for survey data analysis for multistage sample designs. Research Triangle Park, Research Triangle Institute, North Carolina, 1992.

30. SOBAL, J. \& STUNKARD, A. J. Socieconomic status and obesity: a review of the literature. Psychol. Bull., 105:20675,1985

31. TANNER, J. M. Foetus into man: physical growth from conception to maturity. 2nd ed. London, Castlemead Publications, 1989.

32. TADDEI, J. A. A. C. Epidemiologia da obesidade na infância. Pediatria moderrna, 24:111-5, 1993.

33. VICTORA, C. G.; BARROS, F. C.; VAUGHAN, J. P. Epidemilogia da desigualdade: um estudo longitudinal de 6000 crianças brasileiras. São Paulo, Editora Hucitec, 1988.

34. WORLD HEALTH ORGANIZATION. A growth chart for international use in maternal and cchild health care: Guidelines for primary health care personnel. Geneva, World Health Organization, 1978.

35. WORLD HEALTH ORGANIZATION. Energy and protein requirements; report of a joint FAO/WHO/UNU Joint Expert Consulation Technical Report Series, 724. Geneva, Switzerland: World Health Organization, 1985.

36. WORLD HEALTH ORGANIZATION. Use and interpretation of anthropometric indicators of nutritional status. Bull. World Health Organ., 64:929-41, 1986.

37. WORLD HEALTH ORGANIZATION. Diet, nutrition, and the prevention of chronic diseases. Technical Report Series, 797. Geneva, Switzerland: World Health Organization, 1990. 